\title{
Implicit Digital Surfaces in Arbitrary Dimensions
}

\author{
Jean-Luc Toutant ${ }^{1}$, Eric Andres ${ }^{2}$, Gaelle Largeteau-Skapin ${ }^{2}$, and Rita Zrour $^{2}$ \\ 1 Clermont Université, Université d'Auvergne, ISIT, UMR CNRS 6284, BP 10448, \\ F-63000 Clermont-Ferrand, France \\ j-luc.toutant@udamail.fr \\ 2 Université de Poitiers, Laboratoire XLIM, SIC, UMR CNRS 7252, BP 30179, \\ F-86962 Futuroscope Chasseneuil, France \\ \{eric.andres, gaelle.largeteau.skapin,rita.zrour\}@univ-poitiers.fr
}

\begin{abstract}
In this paper we introduce a notion of digital implicit surface in arbitrary dimensions. The digital implicit surface is the result of a morphology inspired digitization of an implicit surface $\left\{\mathbf{x} \in \mathbb{R}^{n}: f(\mathbf{x})=0\right\}$ which is the boundary of a given closed subset of $\mathbb{R}^{n}$, $\left\{\mathbf{x} \in \mathbb{R}^{n}: f(\mathbf{x}) \leq 0\right\}$. Under some constraints, the digital implicit surface has some interesting properties, such as $k$-tunnel freeness. Furthermore, for a large class of the digital implicit surfaces, there exists a very simple analytical characterization.
\end{abstract}

Keywords: Implicit Curve, Implicit Surface, Digital Object, Flake Digitization.

\section{Introduction}

In computer graphics, implicit surfaces $\left\{\mathbf{x} \in \mathbb{R}^{n}: f(\mathbf{x})=0\right\}$ play a fundamental role because of their powerful expressiveness for modeling and their ability to describe general closed manifolds [1, 2]. It is a very convenient way to define surfaces or more generally isosurfaces. The question regarding implicit surfaces in the discrete space is a long standing problem that has been studied mainly because it allows the visualization of often (topologically) complicated surfaces 3, 4]. Different rasterization algorithms for implicit curves and surfaces have been proposed [4 9]. Many of the rasterization methods dealing with implicit curves and surfaces are associated with some subdivision scheme in order to deal with all the singularities and topological inconsistencies that may appear at a given scale. None of the methods however, to the authors best knowledge, have defined a digital implicit surface in arbitrary dimension in a simple mathematical way.

In this paper we address the problem of defining a digital implicit equivalent to an implicit surface in arbitrary dimensions. The rasterization process itself is not addressed although a naive method, consisting in testing all the voxels in a given box, is trivial to implement with our proposed analytical characterization.

E. Barcucci et al. (Eds.): DGCI 2014, LNCS 8668, pp. 332-343 2014.

(c) Springer International Publishing Switzerland 2014 
In the paper we investigate the topological properties of the so defined digital implicit surface and show that we may achieve properties such as $k$-tunnel freeness. Our analytical characterization is however not completely general. We show under which conditions, the analytical characterization is not accurate. This happens mainly when the curvature is large and/or when the surface circumvolution details are small compared to the size of a voxel. Precise criteria on the $r$-regularity of the surface are provided. One of the forthcoming works will consist in subdividing the grid at such places in order to increase the precision and remove the topological errors in the digitization.

The digitization method is a morphology inspired digitization scheme with structuring elements called adjacency flakes. They have been introduced in [10] in order to analytically characterize minimal (with respect to set inclusion) and $k$-separating digital hyperspheres. Using adjacency flakes as structuring elements in the digitization scheme provides the offset region defining the digital object with quite simple analytical characterization, while preserving important topological properties. This allows us to analytically characterize $k$-tunnel free implicit digital surfaces.

In section 2, we will present the digitization models and present a somewhat simplified flake family than the one proposed in [10]. In section 3, we will discuss the conditions under which topological properties are preserved by the digitization process as we propose it. In section 4, we show that, under these conditions, the digital implicit surface can be, correctly and simply, analytically characterized. We conclude this paper, in section 5, with a short discussion and some perspectives.

Now, let us end this introduction with some recalls and notations.

\section{$1.1 \quad$ Recalls and Notations}

Let $\left\{\mathbf{e}_{\mathbf{1}}, \ldots, \mathbf{e}_{\mathbf{n}}\right\}$ be the canonical basis of the $n$-dimensional Euclidean vector space. We denote by $x_{i}$ the $i$-th coordinate of a point, or a vector, $\mathbf{x}$, that is its coordinate associated to $\mathbf{e}_{\mathbf{i}}$. A digital object is a set of integer points. A digital inequality is an inequality with coefficients in $\mathbb{R}$ from which we retain only the integer coordinate solutions. A digital analytical object is a digital object defined by a finite set of digital inequalities.

To each integer point $\mathbf{v}$, a region is associated denoted by $\mathcal{V}(\mathbf{v})$ and called a voxel. It corresponds to the Voronoï cell of $\mathbf{v}$ in the Voronoï partition of the Euclidean space $\mathbb{R}^{n}$, with $\mathbb{Z}^{n}$ as seeds. Geometrically, a voxel is the unit hypercube (ball of radius $1 / 2$ based on the $\ell^{\infty}$-norm) centered on $\mathbf{v}$.

For all $k \in\{0, \ldots, n-1\}$, two integer points $\mathbf{v}$ and $\mathbf{w}$ are said to be $k$-adjacent or $k$-neighbors, if for all $i \in\{1, \ldots, n\},\left|v_{i}-w_{i}\right| \leq 1$ and $\sum_{j=1}^{n}\left|v_{j}-w_{j}\right| \leq n-k$. In the 2-dimensional plane, the 0 - and 1-neighborhood notations correspond respectively to the classical 8- and 4-neighborhood notations. In the 3-dimensional space, the 0-, 1- and 2-neighborhood notations correspond respectively to the classical 26-, 18- and 6-neighborhood notations. 
A $k$-path is a sequence of integer points such that every two consecutive points in the sequence are $k$-adjacent. A digital object $\mathrm{E}$ is $k$-connected if there exists a $k$-path in E between any two points of E. A maximum $k$-connected subset of $\mathrm{E}$ is called a $k$-connected component. Let us suppose that the complement of a digital object $\mathrm{E}, \mathbb{Z}^{n} \backslash \mathrm{E}$, admits exactly two $k$-connected components $\mathrm{C}_{1}$ and $\mathrm{C}_{2}$, or, in other words, that there exists no $k$-path joining integer points of $\mathrm{C}_{1}$ and $\mathrm{C}_{2}$. Then, $\mathrm{E}$ is said to be $k$-separating in $\mathbb{Z}^{n}$.

Let $\oplus$ be the dilation, known as Minkowski addition, such that $\mathcal{A} \oplus \mathcal{B}=$ $\cup_{\mathbf{b} \in \mathcal{B}}\{\mathbf{a}+\mathbf{b}: \mathbf{a} \in \mathcal{A}\}$. Let $\ominus$ be the erosion, such that $\mathcal{A} \ominus \mathcal{B}=\cap_{\mathbf{b} \in \mathcal{B}}\{\mathbf{a}-\mathbf{b}$ : $\mathbf{a} \in \mathcal{A}\}$.

The Gauss digitization, denoted by $\mathbb{G}(\mathcal{E})$, and the Supercover digitization, denoted by $\mathbb{S}(\mathcal{E})$, of a set $\mathcal{E} \subseteq \mathbb{R}^{n}$ are defined as follows:

$$
\begin{aligned}
& \mathbb{G}(\mathcal{E})=\left\{\mathbf{v} \in \mathbb{Z}^{n}: \mathbf{v} \in \mathcal{E}\right\} \\
& \mathbb{S}(\mathcal{E})=\left\{\mathbf{v} \in \mathbb{Z}^{n}: \mathcal{V}(\mathbf{v}) \cap \mathcal{E} \neq \emptyset\right\} .
\end{aligned}
$$

The Gauss digitization is the set of integer points lying in the initial set whereas the Supercover is the set of integer points for which the associated voxel shares at least one point with the initial set.

\section{Digitization Model}

Let us consider a closed subset $\mathcal{E}$ in $\mathbb{R}^{n}(n \geq 2)$. we denote $\partial \mathcal{E}$ the boundary of $\mathcal{E}$. Right now it does not really matter but the aim of course is to suppose that the boundary can be implicitly described by $\left\{\mathbf{x} \in \mathbb{R}^{n}: f(\mathbf{x})=0\right\}$ and the closed set $\mathcal{E}$ by $\left\{\mathbf{x} \in \mathbb{R}^{n}: f(\mathbf{x}) \leq 0\right\}$.

In the sequel of the paper, such a boundary is called a surface $\mathcal{S}=\partial \mathcal{E}$. It induces a partition of $\mathbb{R}^{n}$ in three subsets, the interior of $\mathcal{E}, \mathcal{E}^{o}=\mathcal{E} \backslash \partial \mathcal{E}$, the complement (or exterior) of $\mathcal{E}, \mathcal{E}^{c}=\mathbb{R}^{n} \backslash \mathcal{E}$ and of course $\partial \mathcal{E}$ itself.

\subsection{The Closed Centered Digitization Model}

The Gauss digitization of a surface $\mathcal{S}$ has not, in general, enough integer points to ensure good topological properties such as separation of the space. The discrete points belonging to a continuous straight line, for instance, have no reason to form a connected discrete object: $\left\{\left(x_{1}, x_{2}\right) \in \mathbb{Z}^{n}: a x_{1}+b x_{2}+c=0\right\}$. In order to obtain a digital surface, one first dilates $\mathcal{S}$ with a structuring element to define a region $\mathcal{O}(\mathcal{S})$, located around $\mathcal{S}$ and called offset region. The digitization of $\mathcal{S}$ is then the Gauss digitization of the offset region, i.e. the set of integer coordinate points lying in $\mathcal{O}(\mathcal{S})$.

Used in conjonction with a closed connected structuring element $\mathcal{A}$ symmetric about the origin, we call this digitization scheme the closed centered model and denote it by $\mathrm{D}_{\mathcal{A}}(\mathcal{S})$ :

$$
\mathrm{D}_{\mathcal{A}}(\mathcal{S})=\mathbb{G}\left(\mathcal{O}_{\mathcal{A}}(\mathcal{S})\right)=\mathbb{G}(\mathcal{S} \oplus \mathcal{A})
$$


An equivalent definition of $\mathcal{O}_{\mathcal{A}}(\mathcal{S})$, useful in the sequel of the paper, is:

$$
\mathcal{O}_{\mathcal{A}}(\mathcal{S})=(\mathcal{E} \oplus \mathcal{A}) \backslash(\mathcal{E} \ominus \mathcal{A})
$$

Alternative models have been introduced to overcome some limitations of the closed centered model [10] (open or semi-open models, exterior or interior Gaussian models, etc.). For the sake of clarity, we here only focus on the closed centered model. Many of the properties described in this paper are also verified for those other models.

\section{$2.2 \quad$ Structuring Elements}

The structuring elements we will consider are called adjacency flakes and can be described as the union of a finite number of straight segments centered on the origin.

Definition 1 (Adjacency flakes). Let $n$ be the dimension of the space and $0 \leq k<n$. The minimal $k$-adjacency flake, $\mathrm{F}_{k}(\rho)$ with radius $\rho \in \mathbb{R}^{+}$is defined by:

$$
\mathrm{F}_{k}(\rho)=\left\{\lambda \mathbf{u}: \lambda \in[0, \rho], \mathbf{u} \in\{-1,0,1\}^{n}, \sum_{i=1}^{n}\left|u_{i}\right|=(n-k)\right\} .
$$

Fig. 1 shows the different adjacency flakes in 2- and 3-dimensional spaces.

An important property is that two integer points $\mathbf{v}$ and $\mathbf{w}$ are $k$-adjacent if $\left(\mathbf{v} \oplus \mathrm{F}_{k}(1 / 2)\right) \cap\left(\mathbf{w} \oplus \mathrm{F}_{k}(1 / 2)\right) \neq \emptyset$.

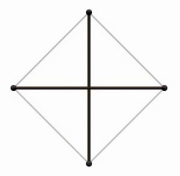

(a) $\mathrm{F}_{1}(\rho)$,

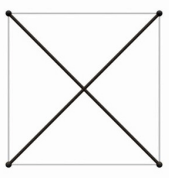

(b) $\mathrm{F}_{0}(\rho)$,

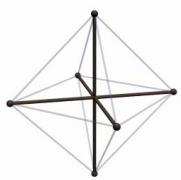

(c) $\mathrm{F}_{2}(\rho)$,

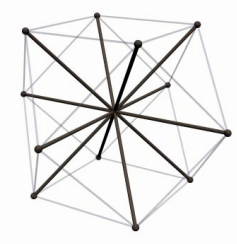

(d) $\mathrm{F}_{1}(\rho)$,

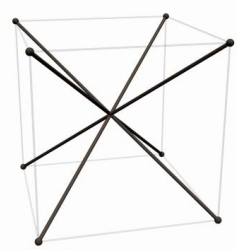

(e) $\mathrm{F}_{0}(\rho)$.

Fig. 1. Adjacency flakes $\mathrm{F}_{1}(\rho), \mathrm{F}_{0}(\rho)$ in the 2-dimensional space and $\mathrm{F}_{2}(\rho), \mathrm{F}_{1}(\rho)$, $\mathrm{F}_{0}(\rho)$ in the 3-dimensional space 
Definition 2. The $\mathrm{F}_{k}$-digitization of a surface $\mathcal{S}$ is the closed centered digitization with structuring element $\mathrm{F}_{k}\left(\frac{1}{2}\right)$ of $\mathcal{S}$. We denote it by $\mathrm{D}_{k}(\mathcal{S})$ and its offset region by $\mathcal{O}_{k}(\mathcal{S})$.

In the sequel of the paper, we only consider the $\mathrm{F}_{k}$-digitizations of surfaces.

\subsection{Digital Implicit Surface}

Now, lets us introduce the definition of digital implicit surface in arbitrary dimensions. We suppose that we deal with an implicit surface $\mathcal{S}=\left\{\mathbf{x} \in \mathbb{R}^{n}: f(\mathbf{x})=0\right\}$ which is the boundary of a closed set $\mathcal{E}$ such that, for all $\mathbf{x} \in \mathcal{E}^{o}$, we have $f(\mathbf{x})>0$ and for all $\mathbf{x} \in \mathcal{E}^{c}$, we have $f(\mathbf{x})<0$.

Definition 3. $A \mathrm{~F}_{k}$-digital implicit surface is the $\mathrm{F}_{k}$-digitization of an implicit surface $\mathcal{S}$.

It is easy to see that that the $\mathrm{F}_{0}$-digital implicit hyperplane corresponds to the supercover of a hyperplane [11] and that the $\mathrm{F}_{0}$-digital implicit hyperspheres are particular cases of the digital hypersperes described in [10]. See Figure 4 for some examples of digital implicit surfaces.

In the next section we are going to examine the topological properties of such surface digitization and in Section 4 we are going to propose an analytical characterization for a large class of digital implicit surfaces.

\section{Preserving Topology}

The purpose of this section is to give conditions on $\mathcal{S}$ to ensure that the digitization preserves some of its topological properties. Ideally, we look for an equivalence between the surface and its digitization, for something close to an homeomorphism. Such a task is out of the scope of the present paper and we restrict our goal to the preservation of the connected components between the complement of the surface and the complement of its digitization. By preservation, we mean that there is a one-to-one correspondence between the connected components of both sets such that each connected component of the second is a proper subset of a unique connected component of the first.

In the sequel, $\mathrm{D}_{k}(\mathcal{S})^{c}$ denotes the complement of $\mathrm{D}_{k}(\mathcal{S})$ in $\mathbb{Z}^{n}$.

First, we study the $k$-tunnel freeness of the $\mathrm{F}_{k}$-digital implicit surfaces to ensure that connected components of the complement are not merged by the digitization process. Then, we focus on conditions to guarantee that none of them disappear or split.

\subsection{Tunnel-Free Digitization}

The notion of $k$-separating set is too restrictive when dealing with surface digitization. In our case, the underlying object $\mathcal{E}$ can be composed of more than one 
connected component and thus the digital analog of its boundary may separate the digital space in more than only two $k$-connected components. The notion of tunnel-free digitization allows to overcome this limitation [12].

Definition 4 (Tunnel-free digitization [12]). A digitization $\mathrm{D}(\mathcal{S})$ of a surface $\mathcal{S}$ is said to be $k$-tunnel-free if for all $\mathbf{v}, \mathbf{w} \in \mathrm{D}(\mathcal{S})^{c}$ such that $\mathbf{v}$ and $\mathbf{w}$ are $k$-adjacent, the straight segment $[\mathbf{v w}]$ does not cross $\mathcal{S}$. If such a couple of voxel exists, it is called a $k$-tunnel of $\mathrm{D}(\mathcal{S})$.

To prove that our digitizations satisfy this property, we first need to introduce the notion of regular set.

Definition 5 (Regular set [13]). Let $\mathcal{E} \subseteq \mathbb{R}^{n}$ be a closed set such that for all $\mathbf{x} \in \partial \mathcal{E}$ it is possible to find two osculating open balls [13] of radius $r$, one lying entirely in $\mathcal{E}^{o}$ and the other lying entirely in $\mathcal{E}^{c}$. Then $\mathcal{E}$ is a r-regular set.

Proposition 1. Let $n$ be the dimension of the space. The $\mathrm{F}_{k}$-digital implicit surface, $\mathrm{D}_{k}(\mathcal{S})$, is a $k$-tunnel-free digitization of $\mathcal{S}=\partial \mathcal{E}$ if $\mathcal{E}$ is a r-regular set with $r>\sqrt{n-k} / 4$.

Proof. Let us consider two integer points $\mathbf{v}, \mathbf{w} k$-adjacent and such that the straight segment $[\mathbf{v w}]$ intersects $\mathcal{S}$ in a point $\mathbf{s}$. if $\mathbf{s}=\mathbf{v}$, then directly $\mathbf{v} \in$ $\mathrm{D}_{k}(\mathcal{S})$. The same occurs for $\mathbf{w}$ if $\mathbf{s}=\mathbf{w}$. In other cases, any open ball of radius $r>\sqrt{n-k} / 4$ through $\mathbf{s}$ (i.e. the center of the ball is at a distance $r$ of $\mathbf{s}$ ) contains at least one point of the union of $k$-adjacency flakes centered at $\mathbf{v}$ and $\mathbf{w}$. Moreover, by definition of $\mathcal{E}$ as a $r$-regular set with $r>\sqrt{n-k} / 4$ (an osculating open ball of radius $r$ entirely in $\mathcal{E}^{o}$ and another one entirely in $\mathcal{E}^{c}$ for each boundary point), it exists a path in $\left(\mathbf{v} \oplus \mathrm{F}_{k}(1 / 2)\right) \cup\left(\mathbf{w} \oplus \mathrm{F}_{k}(1 / 2)\right)$ with one end-point in $\mathcal{E}^{c}$ and the other one in $\mathcal{E}^{o}$. This path necessarily intersects $\mathcal{S}=\partial \mathcal{E}$ in at least one point $\mathbf{s}^{\prime}$. By symmetry of the adjacency flake, either $\mathbf{v}$ or w belongs to $\mathbf{s}^{\prime} \oplus \mathrm{F}_{k}(1 / 2)$ and thus belongs to $\mathrm{D}_{k}(\mathcal{S})$.

It does not exist a couple of $k$-adjacent integer points $(\mathbf{v}, \mathbf{w})$ outside $\mathrm{D}_{k}(\mathcal{S})$ such that the straight segment $[\mathbf{v w}]$ intersects $\mathcal{S}$.

This result means that, under non very restrictive conditions, whatever the supporting surface $\mathcal{S}$, a $k$-connected component of the digital complement of the $\mathrm{F}_{k}$-digital implicit surface $\mathrm{D}_{k}(\mathcal{S})$ only contains points belonging to a unique connected component of $\mathcal{S}^{c}$ : two connected components of the complement of the initial surface cannot be merged by the digitization. Nevertheless, some connected components of $\mathcal{S}^{c}$ may have no representative in $\mathrm{D}_{k}(\mathcal{S})^{c}$ : they can be deleted by the digitization. Or, on the contrary, some may have representatives in several $k$-connected components of $\mathrm{D}_{k}(\mathcal{S})^{c}$ : they can be split by the digitization.

The following part discusses conditions to obtain a one to one correspondence between the connected components of $\mathcal{S}^{c}$ and the $k$-connected components of $\mathrm{D}_{k}(\mathcal{S})^{c}$, i.e. no collapses and no splits occur. 


\subsection{Preserving Connected Components by Digitization}

We work in two steps. We first introduce a condition to ensure that the connected components of $\mathcal{S}^{c}$ are preserved by the dilation. Then, we study the condition ensuring that the Gauss digitization also preserves them.

An immediate result concerns the first step:

Proposition 2. The connected components are preserved between the complement of $\mathcal{S}=\partial \mathcal{E}$ and the complement of $\mathcal{O}_{k}(\mathcal{S})$, if $\mathcal{E}$ is a r-regular set with $r>\sqrt{n-k} / 2$.

Proof. By definition of $\mathcal{E}$ as regular set, any connected component $\mathcal{C}$ of $\mathcal{S}^{c}$ contains at least a closed Euclidean ball of radius $r$ and center c. Since, $\mathrm{F}_{k}(1 / 2) \subset \mathcal{B}(\sqrt{n-k} / 2)$, c is not in $\mathcal{O}_{k}(\mathcal{S})$, and $\mathcal{C} \backslash \mathcal{O}_{k}(\mathcal{S})$ is not empty. $\mathcal{C}$ is itself a connected $r$-regular set with $r>\sqrt{n-k} / 2$. The set resulting of its erosion by $\mathrm{F}_{k}(1 / 2)$ is connected.

The next step is to ensure that the Gauss digitization of each connected components of $\mathcal{O}_{k}(\mathcal{S})^{c}$ is not empty.

First, Lemma 1 gives a sufficient condition on a connected set to ensure that it contains at least one digital point, i.e. it does not collapse. Then, Lemma 2 and Proposition 3 state that the Gauss digitization of such a set is always a $(n-1)$-connected set, i.e. it is not split.

Lemma 1. Let $r>r^{\prime}>\sqrt{n} / 2$. Let $\mathcal{A}$ be a connected $r$-regular set. Let $\mathcal{A}^{\prime}$ be the open interior of the erosion of $\mathcal{A}$ by a closed Euclidean ball of radius $r^{\prime}$. Then, one has $\emptyset \subset \mathbb{S}\left(\mathcal{A}^{\prime}\right) \subset \mathcal{A}$.

Proof. $\mathcal{A}$ is a $r$-regular set, so it contains at least one ball of radius $r$. The center of this ball lies necessarily in $\mathcal{A}^{\prime}$ since $r^{\prime}<r$. It ensures that $\mathcal{A}^{\prime}$ is not reduced to the empty set. Since the supercover of a non empty set is not empty, $\mathbb{S}\left(\mathcal{A}^{\prime}\right)$ contains at least one integer point, or, in other words, $\emptyset \subset \mathbb{S}\left(\mathcal{A}^{\prime}\right)$.

Let us now suppose that $\mathbf{x} \in \mathcal{A}^{\prime}$ and $\mathbf{y} \in \mathbb{R}^{n} \backslash \mathcal{A}$. One has $d(\mathbf{x}, \mathbf{y})>\sqrt{n} / 2$. $\mathbf{x} \in \mathcal{V}(\mathbf{y})$ would imply that $d(\mathbf{x}, \mathbf{y}) \leq \sqrt{n} / 2$. Thus $\mathbf{x}$ belongs necessarily only to voxels with center in $\mathcal{A}$ and one has $\mathbb{S}\left(\mathcal{A}^{\prime}\right) \subset \mathcal{A}$.

Lemma 2. Let $\mathcal{A}$ and $\mathcal{A}^{\prime}$ be defined as in Lemma 1 , Then, $\mathbb{S}\left(\mathcal{A}^{\prime}\right)$ is $(n-1)$ connected.

Proof. $\mathcal{A}$ is a connected $r$-regular set. Thus $\mathcal{A}^{\prime}$ is an open, connected set [13]. The supercover of a connected set is a $(n-1)$-connected set [14].

Proposition 3. Let $\mathcal{A}$ and $\mathcal{A}^{\prime}$ be defined as in Lemma 1, Then, the Gauss digitization of $\mathcal{A}, \mathbb{G}(\mathcal{A})$, is a $(n-1)$-connected set.

Proof. Let $\mathbf{v}$ be any integer point in $\mathcal{A}$. Let $\mathcal{B}(r)$ be the open ball of radius $r$ based on the Euclidean norm. Then there exists a point $\mathbf{c}$ such that $\mathcal{B}(r) \oplus\{\mathbf{c}\} \subseteq \mathcal{A}$ and $\mathbf{v} \in \mathcal{B}(r) \oplus\{\mathbf{c}\}$. c lies in $\mathcal{A}^{\prime}$ and belongs to $\mathcal{V}(\mathbf{w})$ (possibly $\mathbf{v}=\mathbf{w}$ ). Consider the supercover of the segment $[\mathbf{v c}]$. Every integer point in it is in the ball $\mathcal{B}(r) \oplus\{\mathbf{c}\}$. So there exists a $(n-1)$-connected path linking $\mathbf{v}$ and $\mathbf{w}$, thus $(n-1)$-connecting $\mathbf{v}$ to $\mathbb{S}\left(\mathcal{A}^{\prime}\right)$. 
By combining results of Proposition 1, Proposition 2] and Proposition [3, we state the main theorem of this section:

Theorem 1. if $\mathcal{E}$ is a r-regular set with $r>(\sqrt{n-k}+\sqrt{n}) / 2$, then the connected components are preserved between $\mathcal{S}^{c}$ and $\mathrm{D}_{k}(\mathcal{S})^{c}$, according to the $k$ adjacency relationship.

\section{Analytical Characterization of a Digital Implicit Surface}

Let us denote the set of end-points of the segments composing the adjacency flake $\mathrm{F}_{k}(\rho)$ by $V_{k}(\rho)=\left\{\mathbf{x}: \mathbf{x} \in\{-\rho, 0, \rho\}^{n}, \sum_{i=1}^{n}\left|x_{i}\right|=\rho(n-k)\right\}$. The following technical lemma shows that, under the condition of Theorem 1, we only need to consider the end-points of the flake line segments to characterize the offset zone.

Lemma 3. Let $\mathcal{S}$ be a surface satisfying the condition of Theorem 1 Let also $\mathbf{x} \in \mathcal{O}_{k}(\mathcal{S})$. Then, there exists $\mathbf{y}, \mathbf{y}^{\prime} \in\left(\mathbf{x} \oplus V_{k}(1 / 2)\right)$ such that $\mathbf{y} \in \mathcal{E}$ and $\mathbf{y}^{\prime} \in \operatorname{cl}\left(\mathcal{E}^{c}\right)$, where $\operatorname{cl}\left(\mathcal{E}^{c}\right)$ is the closure of $\mathcal{E}^{c}$.

Proof. By definition of $\mathcal{O}_{k}(\mathcal{S}),\left(\mathbf{x} \oplus \mathrm{F}_{k}(1 / 2)\right) \cap \mathcal{S} \neq \emptyset$. Due to the condition of Theorem 1, the number of intersections between a segment of $\mathbf{x} \oplus \mathrm{F}_{k}(1 / 2)$ and $\mathcal{S}$ is lower or equal to 2 . Let us consider a segment of $\mathbf{x} \oplus \mathrm{F}_{k}(1 / 2)$ intersecting $\mathcal{S}$. Either one of its end-points is in $\mathcal{E}$ and the other in $\operatorname{cl}\left(\mathcal{E}^{c}\right)$, or both are in $\mathcal{E}^{o}$ or in $\mathcal{E}^{c}$. In the first case, the result is immediate. In the second case, there is necessarily another segment of $\mathbf{x} \oplus \mathrm{F}_{k}(1 / 2)$ which satisfies the first case.

Figure 2 illustrates the lemma. It shows, on the left, a case where considering only the end-points of the flake is equivalent to considering the whole flake and, on the right, a case where it is not equivalent. This leads immediately to the following theorem which allows a very simple analytical characterization for a large class of implicit digital surfaces:

Theorem 2. Let $\mathcal{S}=\left\{\mathbf{x} \in \mathbb{R}^{n}: f(\mathbf{x})=0\right\}$ be an implicit surface (boundary of a closed set $\mathcal{E}$ such that, for all $\mathbf{x} \in \mathcal{E}^{o}$, we have $f(\mathbf{x})>0$ and for all $\mathbf{x} \in \mathcal{E}^{c}$, we have $f(\mathbf{x})<0$ ) satisfying the condition of Theorem 1 ( $\mathcal{E}$ is a r-regular set with $r>(\sqrt{n-k}+\sqrt{n}) / 2)$. Then, the $\mathrm{F}_{k}$-digital implicit surface $\mathrm{D}_{k}(\mathcal{S})$ is analytically characterized as follows:

$$
\mathrm{D}_{k}(\mathcal{S})=\left\{\mathbf{v} \in \mathbb{Z}^{n}: \begin{array}{r}
\min \left\{f(\mathbf{x}): \mathbf{x} \in\left(\mathbf{v} \oplus V_{k}(1 / 2)\right)\right\} \leq 0 \\
\text { and } \max \left\{f(\mathbf{x}): \mathbf{x} \in\left(\mathbf{v} \oplus V_{k}(1 / 2)\right)\right\} \geq 0
\end{array}\right\} .
$$

Proof. According to Lemma 3 , for any $\mathbf{v} \in \mathrm{D}_{k}(\mathcal{S})$, it exists $\mathbf{x}, \mathbf{x}^{\prime} \in\left(\mathbf{v} \oplus V_{k}(1 / 2)\right)$ such that $\mathbf{x} \in \mathcal{E}$ and $\mathbf{x}^{\prime} \in \operatorname{cl}\left(\mathcal{E}^{c}\right)$. Since for all $\mathbf{x} \in \mathcal{E}^{o}$, we have $f(\mathbf{x})>0$ and for all $\mathbf{x} \in \mathcal{E}^{c}$, we have $f(\mathbf{x})<0$, the analytic formulation is immediate.

A $\mathrm{F}_{k}$-digital implicit surface is thus entirely defined by the knowledge of the function $f$ and of the value $k$. 


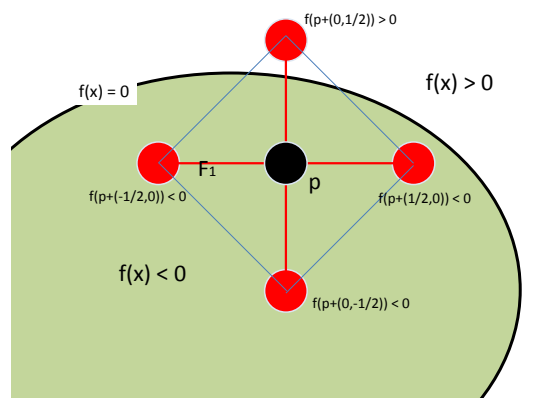

(a) A case where the digitization with only the end-points of the flake is equivalent to the digitization with the whole flake,

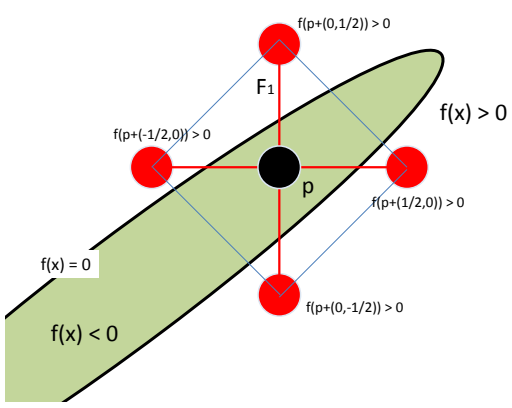

(b) A case where the two digitizations lead to different results.

Fig. 2. Illustration of the limits of the analytical characterization

\section{Discussion, Conclusion and Perspectives}

In the present paper, we have introduced a simple analytical definition of digital implicit surfaces. They are built as the digitizations of an implicit surface $\mathcal{S}=$ $\left\{\mathbf{x} \in \mathbb{R}^{n}: f(\mathbf{x})=0\right\}$ satisfying some specific conditions. Namely, $\mathcal{S}$ should be the boundary of a closed set $\mathcal{E}=\left\{\mathbf{x} \in \mathbb{R}^{n}: f(\mathbf{x}) \leq 0\right\}$ which is $r$-regular with $r>(\sqrt{n-k}+\sqrt{n}) / 2$.

In addition to the analytical characterization, these conditions ensures the $k$ tunnel freeness of the digital implicit surfaces. They also preserve the connected components between the complement of the implicit surface and the complement
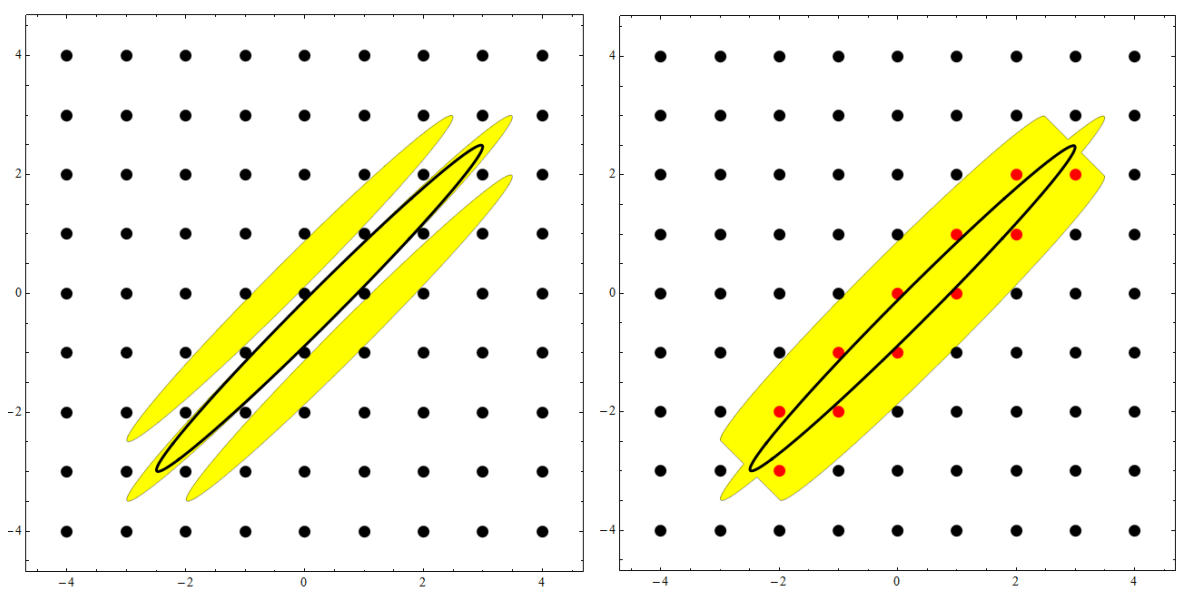

Fig. 3. In yellow, on the left, the offset region obtained by only considering $V_{k}(1 / 2)$ as structuring element, on the right, the real offset region of the two dimensional implicit conic $201 x^{2}-398 x y+201 y^{2}-200 x+200 y+20=0$ 
of its digitization with regard to the $k$-adjacency relationship. These conditions are of course sufficient but not necessary.

Figure 3 presents an extreme case where the analytical characterization fails to correctly represent the $\mathrm{F}_{0}$-digital implicit surface. On the left of the figure, we see that the offset region that we obtain with the analytical characterization is composed by three distinct connected components and contains, in this case, no integer points. According to the analytical characterization, the digitization of the ellipse is an empty set. The Condition of Theorem 11 and more specifically the condition of Proposition 1 (to ensure a tunnel-free digitization), is not met. On the right of the figure, we have the correct result corresponding to the $\mathrm{F}_{0}$-digital implicit ellipse. This is the limit of the analytical characterization proposed in this paper : if the condition of Theorem 1 (or a quite less restrictive, but very similar one) is not met, the proposed analytical characterization is just an approximation of the digital implicit surface, since not only the end-points of the flake line segments contribute to the boundary of the offset zone. It is necessary to compute the intersection between said line segments and the implicit surface. This can be accomplished in several ways. It can be done with help of the derivatives or by direct intersection computation ; however exact computations can only be done for a limited class of surfaces and the result will not be as simple as the one proposed in this paper. Another obvious limitation of the method is that it is limited to $(n-1)$-dimensional surfaces in dimension $n$. It does not work for 3D curves for instance; but intersecting a surface with a flake line segment or intersecting a curve with the faces of an adjacency norm ball is a problem of somewhat similar nature and gives a good, although not simple, way of proposing three-dimensional curves of specific connectivity. We plan to propose such descriptions in a forthcoming paper.

Another problem occurs even in the corrected version of Figure 3 the interior of the ellipse disappeared during the digitization process. The condition of Theorem 1, and more specifically the condition of Proposition 2, is not met. This is inherently a problem of grid size. One way around the problem is to locally refine the grid size. This is not new and is actually the way the digitization of implicit curves and surfaces have been done most of the time [1, 2, 4 9]. Our aim is to explore such subdivision methods for surfaces in dimension $n$. 

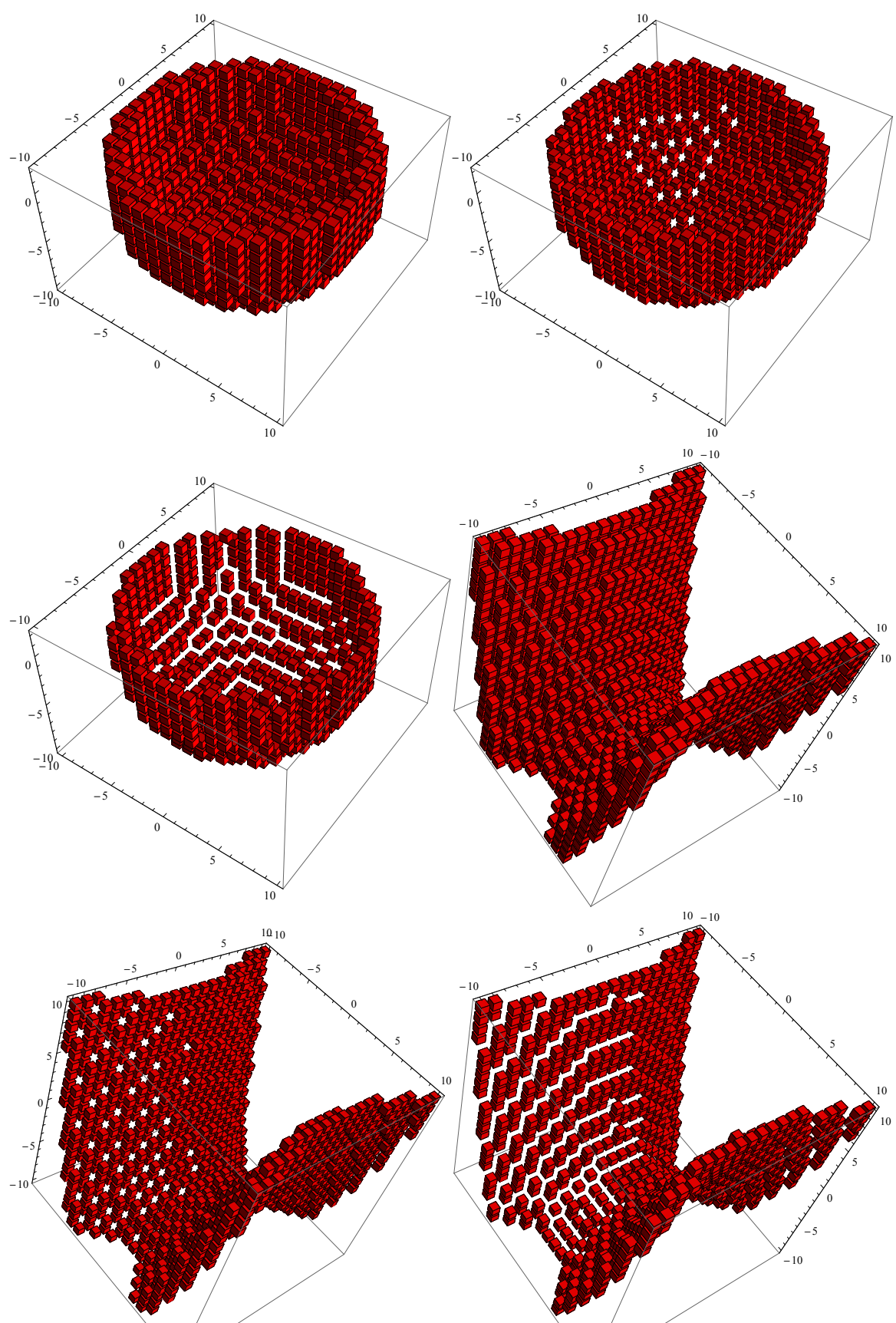

Fig. 4. Examples of digital implicit surface: $\mathrm{F}_{0^{-}}, \mathrm{F}_{1}-$ and $\mathrm{F}_{2}$-digital implicit spheres of radius 9 (cut in order to see the tunnels) and digital implicit quadric $9 x^{2}-4 y^{2}-36 z-$ $180=0$ 


\section{References}

1. Bloomenthal, J., Wyvill, B. (eds.): An Introduction to Implicit Surfaces. Morgan Kaufmann Publishers Inc., San Francisco (1997)

2. Velho, L., Gomes, J., de Figueiredo, L.H. (eds.): Implicit Objects in Computer Graphics. Springer (2002)

3. Stolte, N.: Arbitrary 3D resolution discrete ray tracing of implicit surfaces. In: Andrès, É., Damiand, G., Lienhardt, P. (eds.) DGCI 2005. LNCS, vol. 3429, pp. 414-426. Springer, Heidelberg (2005)

4. Emeliyanenko, P., Berberich, E., Sagraloff, M.: Visualizing arcs of implicit algebraic curves, exactly and fast. In: Bebis, G., et al. (eds.) ISVC 2009, Part I. LNCS, vol. 5875, pp. 608-619. Springer, Heidelberg (2009)

5. Stolte, N., Caubet, R.: Comparison between Different Rasterization Methods for Implicit Surfaces. In: Visualization and Modeling, ch. 10. Academic Press (1997)

6. Stolte, N., Kaufman, A.: Novel techniques for robust voxelization and visualization of implicit surfaces. Graphical Models 63(6), 387-412 (2001)

7. Sigg, C.: Representation and Rendering of Implicit Surface. Phd thesis, diss. eth no. 16664, ETH Zurich, Switzerland (2006)

8. Taubin, G.: An accurate algorithm for rasterizing algebraic curves. In: Proceedings of the 2nd ACM Solid Modeling and Applications, pp. 221-230 (1993)

9. Taubin, G.: Rasterizing algebraic curves and surfaces. IEEE Computer Graphics and Applications 14(2), 14-23 (1994)

10. Toutant, J.-L., Andres, E., Roussillon, T.: Digital circles, spheres and hyperspheres: From morphological models to analytical characterizations and topological properties. Discrete Applied Mathematics 161(16-17), 2662-2677 (2013)

11. Andres, E.: The supercover of an $\mathrm{m}$-flat is a discrete analytical object. Theoretical Computer Science 406(1-2), 8-14 (2008)

12. Cohen-Or, D., Kaufman, A.E.: Fundamentals of surface voxelization. CVGIP: Graphical Model and Image Processing 57(6), 453-461 (1995)

13. Stelldinger, P., Köthe, U.: Towards a general sampling theory for shape preservation. Image and Vision Computing 23(2), 237-248 (2005)

14. Brimkov, V.E., Andres, E., Barneva, R.P.: Object discretizations in higher dimensions. Pattern Recognition Letters 23(6), 623-636 (2002) 